\title{
Business Process Management e information technology infrastructure library no processo de gerenciamento de liberação de versão
}

\author{
Dagr DantedeQiveiraGatto Mestrando em Informática e Gestão do Conhecimento. Universidade Nove de Julho (UNINOVE) - \\ Brasil. dacyr.gatto@uni9.pro.br \\ Echpd BuenoPadbFarias Mestre em Informática e Gestão do Conhecimento. Universidade Nove de Julho (UNINOVE) - Brasil. \\ farias.edquel@uninove.edu.br \\ RentojoéSæri Doutor em Engenharia Elétrica pela Escola Politécnica da Universidade de São Paulo (EPUSP) - \\ Brasil. sassi@uni9.pro.br
}

\begin{abstract}
RESUMO
O processo de Gerenciamento de Liberação de Versão é responsável pela implementação de mudanças no ambiente de infraestrutura de Tecnologia da Informação, através do uso de um conjunto de itens de configuração. Caso ocorra alteração nestes itens de configuração, é necessária a intervenção e reestruturação do processo para eliminar falhas. Em situações como esta se utiliza a metodologia BPM na documentação, manipulação e formalização de processos de negócios. Utiliza ainda o framework ITIL na implementação do gerenciamento de serviços focado em pessoas, processos e recursos utilizados. A empresa desenvolvedora de software abordada neste artigo, apresentou a alteração na distribuição de itens de configuração que fazem parte deste processo causando problemas no processo de Gerenciamento de Liberação de Versão. Sendo necessária a intervenção profissional com o uso da metodologia BPM e do framework ITIL para reestruturação do mesmo e eliminação das falhas. Sendo assim o objetivo deste trabalho foi avaliar a aplicação da metodologia BPM e do framework ITIL no processo de Gerenciamento de Liberação de Versão em uma empresa desenvolvedora de software. Para alcançar este objetivo utilizou-se uma abordagem qualitativa dividida em duas fases: Fase 1, definição e mapeamento do processo de Gerenciamento de Liberação de Versão com a aplicação da metodologia BPM e Fase 2, aplicação do framework ITIL sobre o processo já definido.
\end{abstract}

Palavras-chave: Framework ITIL. Metodologia BPM. Gerenciamento. Liberação de Versão.

\section{Business Process Management and information technology infrastructure library in the version release management process}

\begin{abstract}
The Version Release Management process is responsible for implementing changes in the Information Technology infrastructure environment through the use of a set of configuration items. If there is a change in these configuration items, it is necessary to intervene and restructure the process to eliminate failures. In situations like this the BPM methodology is used in the documentation, manipulation and formalization of business processes. It also uses the ITIL framework in the implementation of service management focused on people, processes and resources used. The software developer company discussed in this article introduced the change in the distribution of configuration items that are part of this process causing problems in the Version Release Management process. Professional intervention is required with the use of the BPM methodology and the ITIL framework for its restructuring and elimination of failures. Therefore, the objective of this work was to evaluate the application of the BPM methodology and the ITIL framework in the Version Release Management process in a software development company. To achieve this goal a qualitative approach was used, divided into two phases: Phase 1, definition and mapping of the Version Release Management process with the application of the BPM methodology and Phase 2, application of the ITIL framew ork on the process al ready defined.
\end{abstract}

Keywords: ITIL Framework, BMP Methodology, Version Release Management Process. 


\section{INTRODUÇÃO}

Dentre os processos gerenciáveis de Tecnologia da Informação (TI), tem-se 0 processo de gerenciamento de liberação de versão, que tem como objetivo construir, testar e entregar serviços capazes de suportar as especificações solicitadas pelo cliente, e assim atingir os objetivos pretendidos.

Para garantir a entrega de serviços de TI e alcançar o resultado esperado pelo cliente, foram desenvolvidos por empresas de pesquisa conjuntos de práticas em gestão de TI buscando melhorar as capacidades em serviços de TI. Dentre estes modelos, ou conjuntos de práticas, pode-se citar o framework ITIL (Information Technology Infraestructure Library) (AXELOS, 2013).

O principal objetivo do ITIL é prover a comunicação entre o negócio e a tecnologia, buscando melhoria contínua dos processos orientada pelo foco no cliente e pela eficácia nos investimentos (LOUREIRO, 2012; GOMES; GOULART JÚNIOR, 2016). De maneira resumida, o ITIL faz referência à garantia dos níveis de serviços acordados com os clientes, sejam eles internos ou externos.

O framework ITIL permite que de acordo com cada contexto, seja possível utilizar outros frameworks ou metodologias para apoiar na compreensão dos processos que serão trabalhados. Entre estes tem-se a metodologia BPM (Business Process Management), que auxilia no mapeamento de processos de negócios, organizando-os para um melhor fluxo da informação, resultando em uma comunicação eficaz entre os envolvidos. Desta forma com o processo mapeado pela metodologia BPM pode-se então visualizar em que atividades o ITIL pode ser aplicada. Silva (2004) preconiza a importância do mapeamento do processo para que então se justifique a correta aplicação do ITIL no referido processo.

Diversos autores corroboram a eficácia e eficiência da aplicação da metodologia BPM em várias áreas de gestão de processos, demonstrados em diversas publicações científicas como segue.

Calazans, Kosloski e Guimarães (2016) aborda a utilização de BPM como um modelo de terceirização de serviços e Pourmirza et al. (2017) que trata de uma revisão da literatura sobre a BPM. Moreira e Silva (2013) em seu estudo apresenta um modelo em que promove o alinhamento entre BPM e ITIL em uma aplicação conjunta para o desenvolvimento de sistemas de informação para gestão financeira.

O ITIL é citado e estudado em diversos aspectos de gestão de processos de TI como demonstrados pelos autores abaixo.

Gil-Gómez, Oltra-Badenes e Adarme-Jaimes (2014) pesquisam a aplicação do ITIL para o gerenciamento de qualidade de serviços assim como Vitoriano e Souza Neto (2016) abordam sobre a maturidade de processos na Administração Federal Brasileira utilizando-se dos padrões descritos no ITIL. Rios, Teixeira Filho e Rios (2017), citam a aplicação do ITIL em Instituições Federais do Ensino Superior, assim como Cruz-Hinojosa e Gutiérrez-de-Mesa (2016), apresentam a aplicação do ITIL em pequenas e médias empresas. Barros e Sassi (2015), realizaram um mapeamento da produção científica nacional e internacional da aplicação do ITIL.

O presente estudo foca na análise da aplicação de BPM alinhada a ITIL, sendo que no caso estudado a equipe de infraestrutura de aplicação da empresa Softplan, alocada no cliente, encontrou problemas na execução do processo para o gerenciamento de liberação de versão de software, e para resolução do mesmo buscou orientações focadas no BPM e ITIL, para entregar uma solução que permitiu que os objetivos da empresa junto ao cliente fossem alcançados.

\subsection{Objetivo Geral}

O objetivo deste estudo foi avaliar a aplicação da metodologia BPM e do framework ITIL no processo de Gerenciamento de Liberação de Versão em uma empresa desenvolvedora de software. 


\subsection{Objetivos Específicos}

Definição e mapeamento do processo de Gerenciamento de Liberação de Versão com a aplicação da metodologia BPM.

Aplicação do frameworkITIL sobre o processo já definido.

\section{FUNDAMENTAÇÃO TEÓRICA} BPM e ITIL.

A seguir são discutidos conceitos do processo de gerenciamento de libração e versão, a metodologia

\subsection{Processo de Gerenciamento de Liberação de Versão}

Conforme definido pelo Great Britain Cabinet Office (2013), o processo de gerenciamento de liberação de versão é responsável pelo processo de implantação das mudanças aprovadas pelo gerenciamento de mudança, sejam estas de TI ou não TI (mudanças de hardware, software, procedimentos, documentos ou pessoas envolvidas no ambiente de TI). Deve-se possuir uma visão sistêmica sobre os serviços de Tl e garantir a proteção do ambiente e de seus serviços através de checagens e procedimentos formais, estruturados e definidos, e que todos os aspectos de uma liberação sejam considerados como um todo. Através deste gerenciamento, 0 ambiente fica protegido contra liberações que não obedecem aos procedimentos formais, pois estabelece mecanismos para isto. Esta visão é discutida por diferentes autores, como Barros e Sassi (2015), onde o mesmo trata de um levantamento sobre a aplicação do ITIL em diversos periódicos, assim como Cruz-Hinojosa e Gutiérrez-de-Mesa (2016) em seu trabalho de revisão da literatura sobre aplicação do ITIL em pequenas e médias empresas, corroborando a importância e abrangência deste assunto ao meio acadêmico.

Dentre os objetivos pretendidos deste processo pode-se descrever (GREAT BRITAIN CABINET OFFICE, 2013): definir e acordar com o cliente as diretrizes para o desenvolvimento de planos de liberação; garantir que cada pacote a ser liberado seja composto por ativos e serviços compatíveis uns com os outros; garantir que a integridade de cada pacote de liberação seja mantida durante todo o processo; garantir que todos os pacotes possam ser rastreados, instalados, testados, verificados e desinstalados, se for o caso; registrar e gerenciar desvios, riscos e questões relacionadas com os serviços novos ou alterados; certificar que há transferência de conhecimento para permitir que o cliente e usuários possam otimizar os usos dos serviços para apoiar as atividades de seus negócios; assegurar que as habilidades e o conhecimento necessários sejam transferidos para as operações e pessoal de apoio, para permitir manter de forma eficaz e eficiente a entrega, a manutenção, e manter os serviços de acordo com as garantias exigidas.

\subsection{Metodologia BPM}

O processo de negócio pode ser definido como uma ordenação de atividades de trabalho onde se utiliza espaço e tempo, juntamente com um conjunto de entradas e saídas, ou seja, é um conjunto de atividades que devem ser executados em uma determinada ordem, onde pode ser executada em paralelo ou sequencialmente. Esta metodologia é amplamente aplicada em diversos setores, como apresentado por Leite, Shibao e Faria (2018) em uma aplicação voltada para processos de negócios verdes (Green BPM), e por Barros e Sassi (2015) aplicada em uma empresa de call-center, onde obteve resultados alinhados ao planejamento estratégico da organização, obtendo melhorias no lucro, no aumento da produção, assim como redução de custos e desperdícios.

Sincorá et al. (2014) apontam em seu estudo vantagens significativas na modelagem de processo de negócio como um conjunto de conceitos, técnicas e modelos que tem por objetivo desenvolver e mapear o modelo de negócio da organização. 0 modelo elaborado é o resultado obtido pela organização, após o 
levantamento de informações relevantes, favorecendo com que o negócio venha a ser entendido por completo. Tem por objetivo mapear os processos existentes, permitindo a identificação de problemas e melhorias que podem ser realizadas. Auxilia a organização a compreender o seu negócio de forma clara. É uma abordagem disciplinada para identificar, desenhar, executar, documentar, medir, monitorar, controlar e melhorar processos automatizados ou não, a fim de alcançar os resultados pretendidos, consistentes e alinhados com as metas estratégicas de uma organização.

Os benefícios que se pode ter na utilização de BPM nas organizações são, ainda de acordo com Sincorá et al. (2014), melhorar o desempenho do negócio através do seu entendimento; simular novas formas para atender o negócio; apoiar a organização em relação às oscilações do mercado; maior controle da duração dos processos representação visual dos processos e dos elementos que os compõem.

\section{$2.3 \mathrm{ITIL}$}

O framework ITIL é um modelo aberto de conjunto de diretrizes de práticas recomendadas, que tem como objetivo ajustar as pessoas, os processos e a tecnologia visando o aumento da eficiência do gerenciamento de serviços. Atualmente a propriedade do framework ITIL pertence a AXELOS, joint venture criada em 2013 pelo OGC e o Capita, empresa internacional de terceirização de processos de negócios, sediada em Londres. A melhoria no processo citado foi obtida a partir de pesquisas realizadas por consultores, especialistas e doutores, para desenvolver as práticas para a gestão da área de TI nas empresas (AXELOS, 2013).

Anteriormente, os processos que envolviam a área de TI eram direcionados para a solução de problemas técnicos pontuais - geralmente internos à organização. Contudo, devido ao acirramento da competitividade em um mundo cada vez mais globalizado, usuários e clientes têm abandonado esse paradigma e vêm concentrando esforços para geração de valor dos negócios, lançando mão dos avanços tecnológicos recentes para implementar processos eficientes, efetivos e econômicos no gerenciamento de recursos (FERNANDES; ABREU, 2014).

Com o avanço da tecnologia, a área de TI passa a ocupar um lugar estratégico nas organizações. Gomes e Goulart Júnior (2016) apresenta em seu trabalho de pesquisa que o framework ITIL promove o alinhamento estratégico da TI com o modelo de negócios da organização, gerando valor, reduzindo custos ou criando novas oportunidades para o negócio.

O uso do ITIL torna os serviços de TI cada vez mais alinhados ao cliente, pois os prazos estabelecidos e a melhoria da qualidade estão diretamente relacionados com a melhoria do relacionamento com o cliente. Além disso, os serviços prestados são descritos de forma mais clara tornando maior o entendimento entre os profissionais de TI e o cliente.

Barros e Sassi (2015) no levantamento de produções científicas sobre a aplicação do ITIL em periódicos apontam que dentre as principais razões para a adoção do framework ITIL estão: Modelo não proprietário: pode ser utilizado por qualquer empresa independente de plataforma tecnológica; Não é um modelo prescritivo: é um modelo flexível, ou seja, deve ser adotado e adaptado além de não depender do tamanho da empresa ou do setor. Fornece práticas já validadas no mercado: as empresas se beneficiam dessa forma não tendo que investir tempo para "reinventar a roda". Usada por milhares de empresas no mundo: ajuda a estabelecer uma terminologia comum entre os provedores internos e externos de Tl.

Segue uma relação de benefícios notórios que podem ser considerados quando implementado o ITIL em uma organização: adotar práticas já homologadas, onde acarreta ganho de tempo; retorno mais dinâmico sobre o projeto de implementação; melhorar a qualidade dos serviços em relação a usuários e clientes; alinhar os serviços de TI com as necessidades da organização, sejam atuais e futuras; aumentar a satisfação do cliente; manter a equipe motivada e focada; os processos se tornarão mais eficientes e eficazes; ter visão mais clara e compreensão da capacidade atual.

Para ser compreendido o relacionamento entre o gerenciamento dos processos de negócio, utilizando-se a metodologia BPM e os serviços de TI, baseados nas práticas framework ITIL, é necessário a definição do processo a ser modelado, onde assim poderão ser identificados os recursos de TI utilizados, assim como as atividades componentes deste processo. 0 alinhamento entre o negócio e a área de TI possibilita, 
também, a definição de critérios para a entrega do resultado gerado pelo processo, proporcionando melhoria na comunicação, no gerenciamento de ativos e atividades profissionais desempenhadas (SILVA, 2004).

\section{METODOLOGIA DE PESQUISA}

Neste capítulo são caracterizadas a pesquisa, a empresa, o problema e a proposta de melhoria.

\subsection{Caracterização Metodológica}

Com relação aos seus objetivos, o presente estudo possui uma abordagem bibliográfica, documental e exploratória visto que se fundamenta no estudo e na interpretação de livros, teses, artigos, documentos técnicos, manuais, dentre outros (GIL, 2008). Com relação a sua abordagem, este estudo foi de natureza qualitativa com caráter descritivo e exploratório em que o ambiente de estudo foi utilizado com fonte direta dos dados levantados, com a finalidade de descrever sistematicamente a situação e o problema encontrado e investigar as possibilidades, buscando esclarecer os conceitos teóricos apresentados no referencial (WAZLAWICK, 2010).

Para o desenvolvimento deste estudo buscou-se materiais referentes à BPM e ao ITIL oriundos de periódicos, congressos e obras para apoiar na contextualização do conteúdo apresentado. Este material bibliográfico foi extraído das bases de conhecimento da Scielo, Science Direct e ResearchGate, e dos Anais de Congressos, sendo que as obras utilizadas são de autores relacionados ao referencial teórico da pesquisa.

Utilizou-se ainda uma análise documental em que se estudou uma empresa desenvolvedora de software, observando o ambiente, acompanhando os membros da equipe nas situações investigadas, efetuando anotações a respeito do comportamento observado, assim como seus resultados, através do acompanhamento das atividades entre abril de 2013 e abril de 2014. Dentre outros cuidados foi solicitada à empresa, autorização formal para a utilização do nome da mesma, assim como dos dados apresentados neste trabalho, a qual forneceu autorização para sua exploração, reconhecendo a importância desta pesquisa.

A realização dos experimentos foi dividida em duas fases: A Fase 1 focou na aplicação da metodologia BPM para identificar e definir o processo de gerenciamento de liberação de versão, definindo atores, atividades, inclusive as atividades críticas do processo. A Fase 2 focou na aplicação do ITIL, onde foram selecionadas ferramentas propostas no framework, como Catálogo de Serviços, Controle de Itens de Configuração, Matriz de Responsabilidades e Key Performance Indicators(KPI). A Figura 1 ilustra a aplicação da metodologia BPM e do frameworkITIL no processo de gerenciamento de liberação de versão em uma empresa desenvolvedora de software.

Figura 1 - Sequência das ações propostas de melhorias

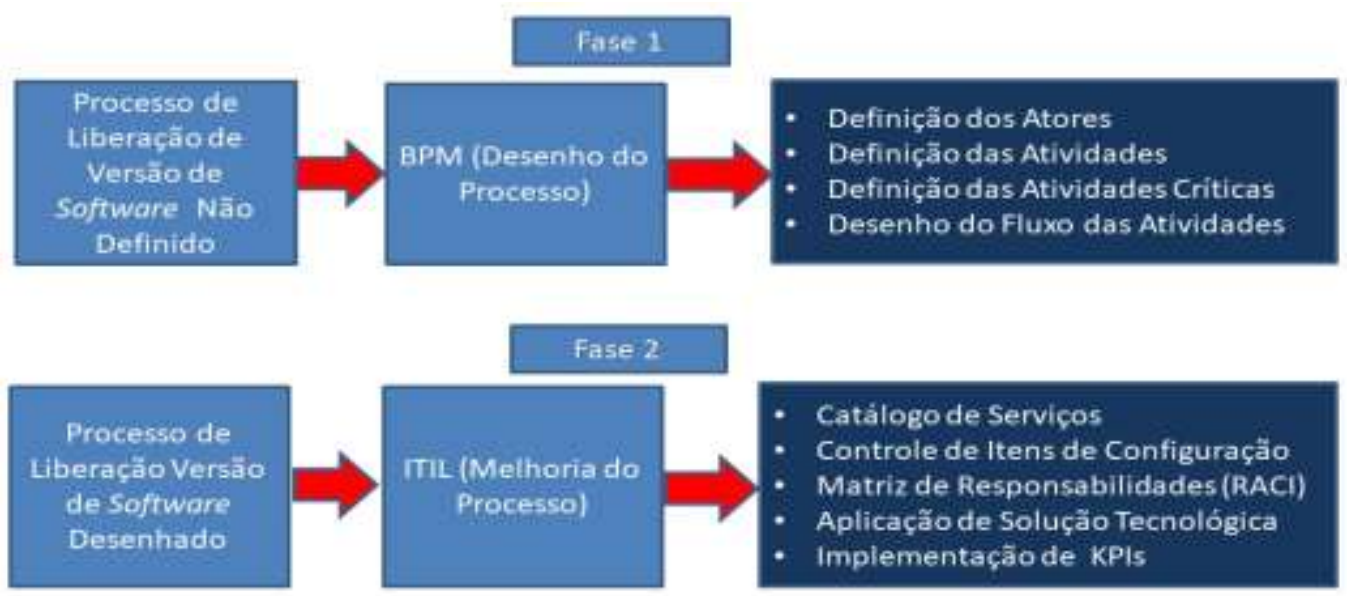

Fonte: Autores (2018). 


\subsection{Caracterização da Empresa}

A empresa Softplan, foco de estudo, é uma desenvolvedora de software, situada na região sul do Brasil, a qual fornece suas soluções para clientes no Brasil, América Latina e Estados Unidos. Atuante desde 1990 em vários segmentos de mercado tais como: Justiça, Infraestrutura e Obras, Gestão Pública, Projetos Cofinanciados por Organismos Internacionais e Indústria da Construção. A Softplan possui um contingente de mais de 1.500 colaboradores atuantes em suas sedes regionais e alocados em seus clientes (SOFTPLAN, 2017).

A atenção deste estudo foi voltada às aplicações da área de automação judiciária, entregue ao seu cliente de São Paulo, onde o número de usuários excede aos 70.000. 0 estudo mostrou o problema que a equipe de infraestrutura de aplicação, alocada no cliente, encontrou na execução do processo para 0 gerenciamento de liberação de versão de software, na busca de orientações focadas no BPM e ITIL, e na solução encontrada para que os objetivos da empresa junto ao cliente fossem alcançados.

A atividade core da área de infraestrutura de aplicação desta empresa consiste na atualização do conjunto dos principais sistemas de automação do seu principal cliente. Quando a aplicação foi fornecida a este cliente, o parque de servidores que compunham a infraestrutura desta aplicação era pequeno e facilmente controlado por um único colaborador que detinha para si o conhecimento dos procedimentos para atualização deste ambiente, além de que toda a atividade era feita de forma manual. No Quadro 1 são relacionados os tipos de servidores da infraestrutura do cliente, que deveriam ser atualizados em cada atividade.

Quadro 1 - Componentes da Infraestrutura do Cliente

\begin{tabular}{|c|l|}
\hline Tipo de Servidor & \multicolumn{1}{|c|}{ Principal Função do Tipo de Servidor } \\
\hline Servidores de Ap licação & $\begin{array}{l}\text { Servidores de grande porte, localizados em Datacenters, responsáveis pela } \\
\text { conexão da aplicação localizada nas estações de trabalho com os Servidores } \\
\text { de Bancos de Dados. }\end{array}$ \\
\hline Servidores de Borda & $\begin{array}{l}\text { Servidores de pequeno porte situados nas localidades físicas do c clien te } \\
\text { servin do como repositório para distribuição dos pacotes de liberação. }\end{array}$ \\
\hline $\begin{array}{c}\text { Servidores de Banco de } \\
\text { Dados }\end{array}$ & $\begin{array}{l}\text { Servidores onde as instâncias de banco de dados são configuradas e } \\
\text { executadas. }\end{array}$ \\
\hline
\end{tabular}

Fonte: Autores (2018)

A aplicação funciona tendo seus objetos (executáveis, bibliotecas, arquivos de ajuda, entre outros) distribuídos entre os Servidores de Aplicação e os Servidores de Borda. A definição de Servidores de Aplicação resume-se a servidores de grande porte, alocados em Datacenters que recebem os objetos denominados Server com a função de manterem a conexão da aplicação da estação do usuário com o banco de dados durante todas as transações. Já o conceito dos servidores de borda funciona como um servidor central na localidade física das estações dos usuários para quando houver atualização de versão, estas estações recebem os objetos denominados Client de forma rápida diretamente do servidor de borda localizado na mesma rede local, sem onerar a infraestrutura da localidade, como por exemplo, sobrecarregar o link de dados na transmissão dos objetos para todas as estações de trabalho.

\subsection{Caracterização do Problema}

Com o advento de um projeto de grande porte objetivando a expansão do ambiente de infraestrutura das aplicações e consequentemente da oferta do conjunto destas aplicações para todos os usuários do Estado de São Paulo deste cliente, a topologia das máquinas (servidores de bancos de dados, servidores de aplicação, servidores de borda) expandiu rapidamente, o que ampliou o escopo da atividade de atualização do conjunto de aplicações tornando o controle vigente no cenário inicial totalmente ineficaz e impraticável pelo único colaborador atuante na área. 0 processo de gerenciamento de liberação de versão, que era feito 
manualmente, não era definido e tornou-se impreciso e ineficaz para atender à necessidade do cliente e do negócio.

Em termos de quantidades o cliente possuía, no período da pesquisa, 197 servidores de aplicação e 507 servidores de borda, que durante o processo de gerenciamento de liberação de versão devem ser atualizados com os objetos da versão e estarem prontos para uso assim que finalizado o procedimento. Também fazem parte 21 servidores de banco de dados, onde scripts de configuração de parâmetros e tabelas são executados para que os bancos de dados se adequem a cada versão de software. Na Figura 2 é representada a topologia do ambiente da aplicação, ilustrando de forma simplificada a comunicação entre eles.

Figura 2 - Representação da Topologia do Ambiente da Aplicação

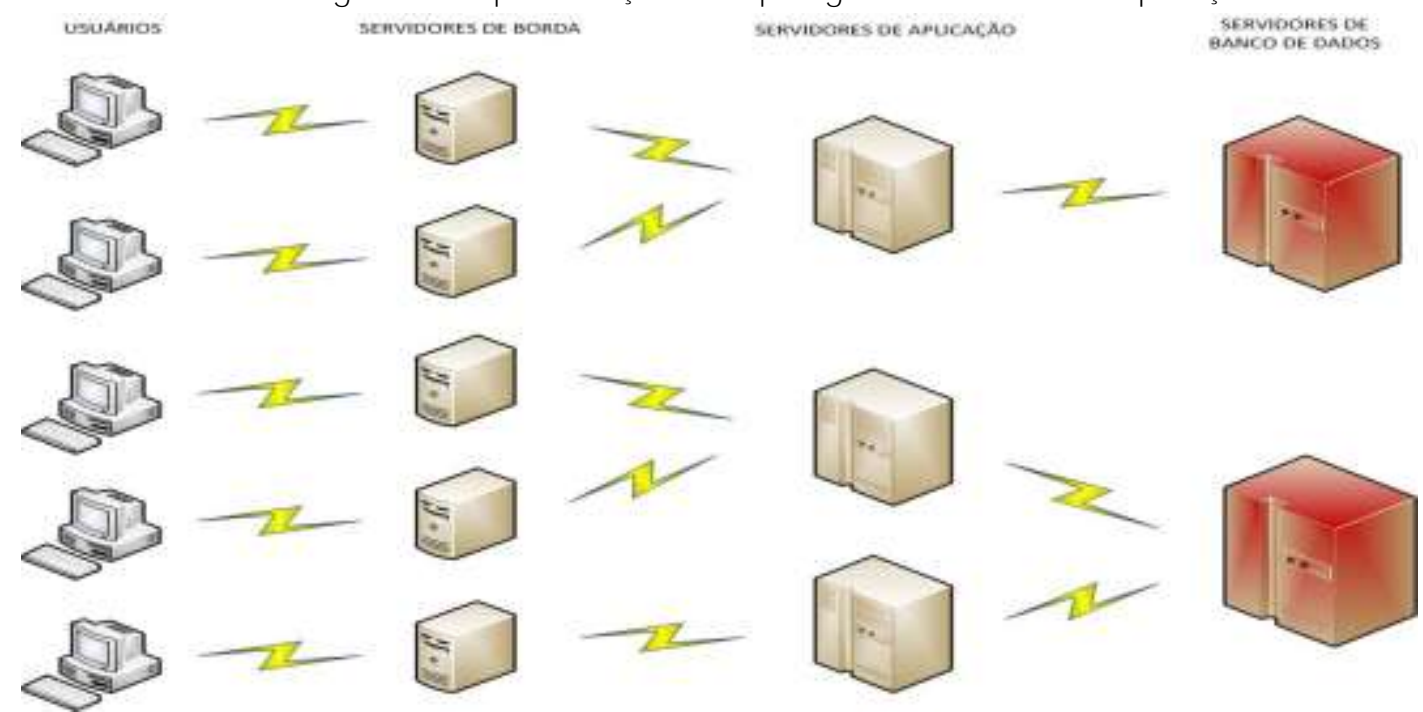

Fonte: Softplan (2017).

Anteriormente, quando o parque de servidores era composto de poucas máquinas, o trabalho de atualização por ser feito manualmente, máquina a máquina atendia as expectativas, porém como mencionado anteriormente, o parque de servidores tornou-se amplo e a distribuição destes objetos e execução de scripts não possuíam nenhum critério documentado, ou processo definido e era executado por um colaborador que apesar de ter conhecimento técnico, se perdia no gerenciamento da atividade, pelo aumento contínuo da quantidade de servidores que passaram a fazer parte da topologia.

Sempre quando o expediente no cliente iniciava o resultado era caótico, onde vários servidores de aplicação e de borda não estavam atualizados com a versão correta, e assim não permitia a atualização de diversas estações de trabalho; scripts executados com erro ou não executados, impedindo vários usuários de executarem suas atividades, uma vez que a aplicação não funcionava de forma adequada, quando muitas vezes nem inicializava.

Como resultado inúmeras falhas de atualização de software eram identificadas assim que o cliente iniciava suas atividades, causando transtorno na principal atividade do cliente que era apoiada pelo conjunto destas aplicações, além de transtorno para a empresa Softplan que tinha que focar emergencialmente nas correções, além das atividades cotidianas da área de infraestrutura de aplicação.

Sendo assim a equipe que acompanhava o início do expediente tinha que identificar e atualizar cada servidor de forma manual e pontual, avaliar se algum script havia falhado em sua execução, o que levava quase um dia inteiro, quando não mais, causando um impacto negativo no cliente e também nas demais atividades diárias da equipe.

Diante deste cenário, sempre que havia a liberação de versão em produção, constantemente esta situação se repetia, causando enorme transtorno, para todas as partes interessadas do cliente e da empresa Softplan. 
Buscando compreender a relação da infraestrutura que compõe o ambiente das aplicações, foram coletadas informações técnicas e de procedimentos que impactam ou influenciam os processos analisados no estudo, através de análise documental e observação das atividades.

Para compreender passo a passo como era executado o processo de gerenciamento de liberação de versão, segue como as atividades eram realizadas:

a) Apenas um analista da equipe ficava responsável por todas as transferências de objetos para todos os servidores, fossem estes de aplicação ou de borda;

b) As transferências eram executadas utilizando um arquivo de lote para cada tipo de servidor com os endereçamentos IP de cada máquina. Assim que este arquivo era executado era iniciada a transferência de servidor por servidor, porém não era confirmado se a transferência havia sido executada com êxito;

c) Sempre novos servidores eram acrescentados ao parque pelo cliente, uma vez que o projeto de expansão estava em execução, porém não se tinha um controle destes servidores por parte da equipe de infraestrutura de aplicação, o que acarretava em vários casos de servidores desatualizados em cada atividade de atualização, pois estes não eram mapeados a tempo no arquivo de lote;

d) Vários servidores de borda eram desligados pelo cliente ao término do expediente de cada localidade, 0 que impedia a atualização destes durante a atividade;

e) Scripts não eram executados de forma correta, ou não eram executados, fazendo com que faltassem as parametrizações novas em alguns servidores de banco de dados.

O cenário que se formou então passou a necessitar de intervenção, monitoramento, controle e mensuração em relação ao gerenciamento de liberação de versão, visando-se restaurar o controle e efetivamente criar um processo definido e gerenciável para que esta atividade fosse executada com o máximo de precisão em todas as suas atividades componentes, sempre que necessário.

Para que a versão fosse distribuída e liberada com sucesso, a atividade dependia da execução das seguintes etapas:

a) Parar os serviços da aplicação nos Servidores de Aplicação;

b) Apagar uma estrutura definida de pastas dentro de cada servidor de aplicação, onde arquivos da versão antiga não seriam mais úteis, e que se continuassem nos servidores, poderiam causar conflito com a versão nova;

c) Transferir os objetos direcionados aos servidores de aplicação que consistiam em executáveis, bibliotecas, documentos informativos entre outros;

d) Transferir os objetos direcionados aos servidores de borda, assim como os de aplicação, cada qual com sua particularidade;

e) Configurar parâmetros do sistema;

f) Executar os Scripts de Banco de Dados;

g) Subir os serviços da aplicação nos servidores de aplicação;

h) Testar a conectividade de cada aplicação.

O cumprimento destas etapas foi essencial para o perfeito funcionamento do sistema. Para alcançar este objetivo a empresa tomou ações buscando apoio na BPM, efetuando um levantamento de como identificar as deficiências em todas as atividades componentes do processo, assim como acompanhar a formalização deste que controlaria as atividades, e assim com o processo mapeado, aplicar o ITIL, focado no processo de gerenciamento de liberação de versão para obter resultados mensuráveis e precisos, entregando ao cliente um serviço de qualidade e valor.

\subsection{Proposta de Melhoria}

A empresa então iniciou um trabalho de reestruturação de colaboradores, processos e tecnologias, buscando orientação na metodologia BPM e no framework ITIL, definindo o fluxo do processo de gerenciamento de liberação de versão, e então com este definido aplicar as práticas propostas no ITIL.

Foi-se nomeado um Coordenador, o qual era certificado ITIL, e este escolheu colaboradores também certificados ITIL, para que então fossem pesquisados neste framework, quais práticas e ferramentas se 
adequariam a realidade que a área de infraestrutura vivia naquele momento. 0 conhecimento destes profissionais foi fundamental para o início desta restruturação, para que pudessem aplicar conceitos sugeridos na BPM e no ITIL e então mapear e organizar o processo de gerenciamento de liberação de versão. Com a definição dos colaboradores que passaram a formar a equipe, foram efetuados levantamentos do nível de conhecimento de cada um nas metodologias que seriam aplicadas no processo sendo estes relacionados no Quadro 2, com todos eles certificados no framework ITIL e com conhecimentos na metodologia BPM.

Quadro 2 - Relação de Colaboradores Certificados em ITIL

\begin{tabular}{|c|c|c|c|}
\hline № de Colaboradores & Função Ocupada & Certificação ITIL & Conhecimento em BPM \\
\hline 1 & Coordenador & Fundamental & Experiente \\
\hline 1 & Analista de Infra Pleno (II) & Intermediário & Intermediário \\
\hline 2 & Analista de Infra Junior (I) & Fundamental & Intermediário \\
\hline
\end{tabular}

Fonte: Autores (2018).

Analisando-se o cenário pode-se verificar a necessidade de se implementar práticas que coordenassem as etapas do processo desde a sua estratégia, desenho, transição e operação de forma a chegarem a um resultado gerenciável e mensurável.

Utilizando o framework ITIL, focando no processo de gerenciamento de liberação de versão, buscaram-se orientações de como organizar o processo. 0 ITIL por ser um framework estrutural, permite que outras técnicas e processos de outros frameworks possam ser utilizados em conjunto. Isto permitiu que técnicas da metodologia BPM fossem utilizadas para o mapeamento do processo e posteriormente de suas atividades componentes.

Foram estas as melhorias propostas:

a) Definir o fluxo de processo, utilizando-se a metodologia BPM, onde ficaram claras as atividades a serem distribuídas a cada membro da equipe, assim como o fluxo a ser seguido, para que cada etapa do processo ficasse bem destacada;

b) Elaborar um Catálogo de Serviços, onde ficassem claras quais eram as atividades da equipe, e onde se deviam concentrar as atenções para o aperfeiçoamento das técnicas e quais processos deveriam ter maior atenção. Neste catálogo seriam relacionadas as atividades que a equipe seria responsável, entre elas a de gerenciamento de liberação de versão;

c) Criar um Controle de Itens de Configuração, onde todos os Servidores (Aplicação, Bordas e Banco de Dados) foram mapeados, e as informações fossem atualizadas praticamente em tempo real. Centralizar todas as informações de to dos os servidores (IPs, hostnames, ranges, endereços físicos das localidades etc.) em uma planilha na nuvem (onde todas as partes interessadas poderão ter acesso às informações de maneira constante);

d) Definir-se as responsabilidades: neste processo haverá de ter um responsável pela Liberação e Implementação de cada tipo de Servidor (Borda e Aplicação); um responsável pela execução de Scripts nos servidores de Bancos de Dados; um responsável pela elaboração de um catálogo de Itens de Configuração, onde estariam documentados e cadastrados cada Servidor de Aplicação, de Borda e de Banco de Dados que faziam parte do parque de servidores, cada qual segundo seu conhecimento e expertise no assunto e no framework. Elaborar uma matriz de responsabilidades (Matriz RACl), que permitirá a definição os responsáveis, prestadores de contas, consultados e informados, durante todo o processo;

e) Definir estratégias de execução de como as atividades de liberação seriam executadas de forma coordenada e contínua entre os novos responsáveis pelas tarefas, e após a conclusão desta a mesma será revisada, de forma a eliminar erros de execução. Os fluxos criados com o auxílio da metodologia BPM permitiram a visualização necessária para a criação das estratégias;

f) Implementar uma ferramenta com o objetivo de distribuição dos Servidores de Borda de forma automática, gerando logs de assertividade, e indicando quais servidores falharam, podendo-se assim gerar um plano de contingência para a atualização destes, antes da liberação em produção. 0 software conhecido internamente como "Console" foi elaborado pela equipe de desenvolvimento da empresa, para substituir os 
arquivos de lote, de primeiro momento, dos Servidores de Borda, para efetuar a distribuição dos objetos de forma simultânea para todas as localidades.

g) Implementar Indicadores-chave de desempenho (Key Performance Indicators - KPIS), que foram estipulados para mensurar o nível de assertividade do processo. Consequentemente estes indicadores determinaram se os objetivos esperados foram alcançados, ou se serão necessárias outras medidas para alcançá-los. No Quadro 3 é possível observar algumas das KPIs aplicadas.

Quadro 3 - KPIs propostos para o Processo de Gerenciamento de Versão

\begin{tabular}{|c|c|}
\hline KPI & Resultado esperado \\
\hline $\begin{array}{l}\text { Número de mudanças implementadas com } \\
\text { sucesso. }\end{array}$ & $\begin{array}{l}\text { Contabilizar quais liberações foram executadas com } \\
\text { sucesso. }\end{array}$ \\
\hline Número de servidores com falhas de atualização. & $\begin{array}{l}\text { Contabilizar quais servidores falharam durante a liberação } \\
\text { de versão. }\end{array}$ \\
\hline Número de scriptscom falhas de execução. & $\begin{array}{l}\text { Identificar e corrigir scripts com falhas, antes de finalizar o } \\
\text { processo. }\end{array}$ \\
\hline Complexidade da versão. & $\begin{array}{l}\text { Mensurar a complexidade da versão através de suas } \\
\text { características, } n \text { - de objetos e no de scripts. }\end{array}$ \\
\hline
\end{tabular}

Fonte: Autores (2018).

\section{APRESENTAÇÃO E DISCUSSÃO DOS RESULTADOS}

Segundo descrito na seção 3 Metodologia de pesquisa, a realização dos experimentos foi feita em duas fases. A seguir são descritas as fases de realização dos experimentos.

\subsection{Fase 1: Aplicação da metodologia BPM}

Com a elaboração do fluxo do processo foi possível definir as atividades, onde situações de contorno seriam aplicadas, para direcionar a atualização das estações de trabalho para servidores de borda secundários, até que os servidores principais fossem recuperados e liberados para atualização, isto antes que liberação da versão fosse finalizada e disponibilizada para o cliente.

Foi utilizado um software de mercado gratuito para auxiliar na elaboração da parte visual e estrutural do processo de gerenciamento de liberação de versão, onde os conceitos de BPM puderam ser aplicados com facilidade pela equipe, a qual já detinha o conhecimento teórico da metodologia. Abaixo segue representado na Figura 3 o fluxo final do processo de gerenciamento de liberação de versão com o detalhamento dos autores, atividades, e atividades críticas devidamente mapeadas, assim como as entradas e saídas do processo, as quais então puderam ser identificadas com clareza. 
Figura 3 - Recorte do Processo de Gerenciamento de Liberação de Versão em BPM.

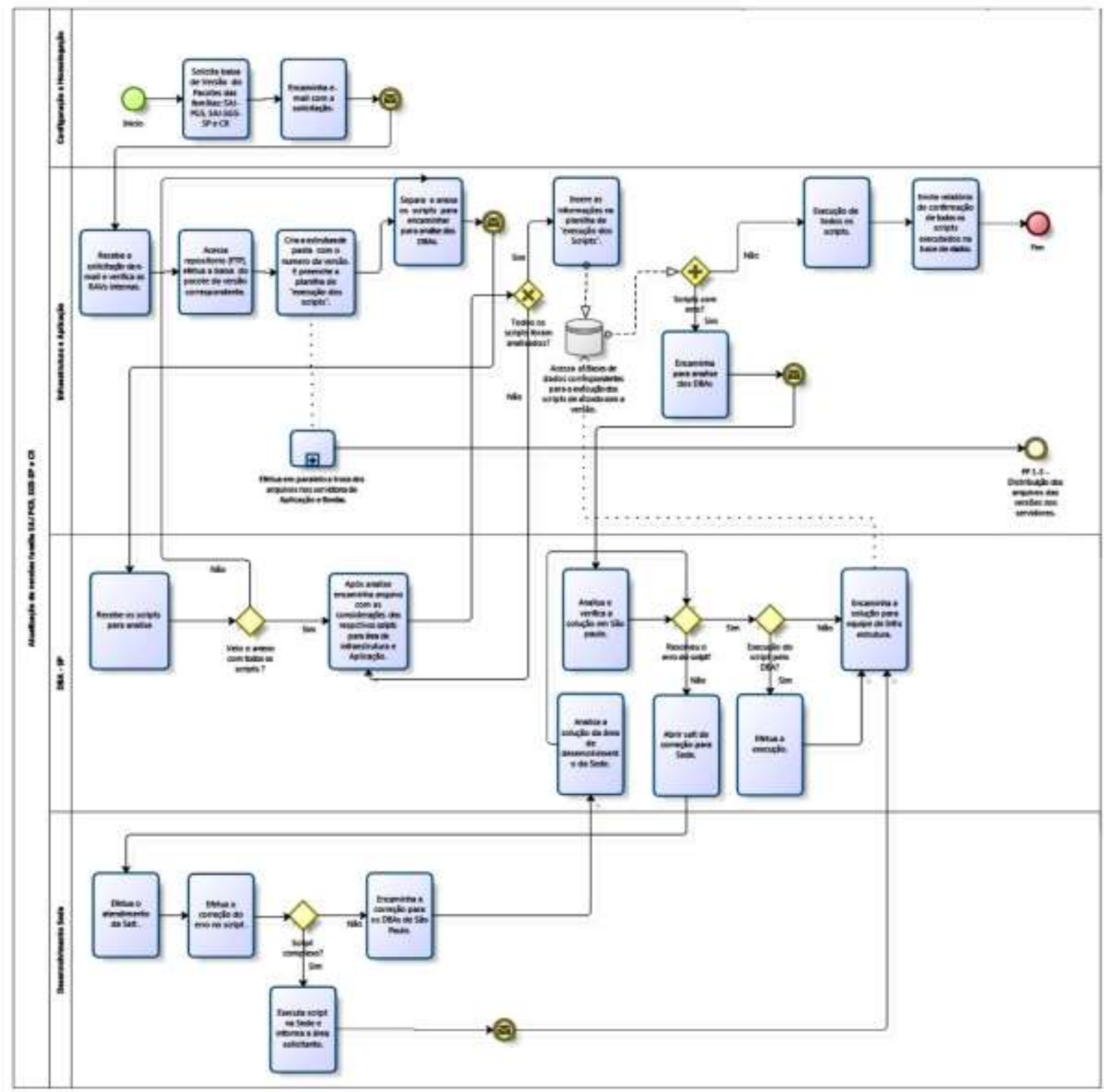

Fonte: Softplan (2017).

\subsection{Fase 1: Aplicação do ITIL}

A elaboração do Catálogo de Serviços, demonstrado na Figura 4, permitiu além de visualizar as atribuições principais da equipe responsável pelo processo de gerenciamento de liberação de versão, também permitiu categorizar as demais atividades executadas pela equipe em todo o seu período de trabalho dentro do cliente, podendo visualizar ações de apoio para a empresa em outras atividades, quando o processo de liberação não era executado, proporcionando novas competências a equipe. 
Figura 4 - Recorte do Catálogo de Serviços

\begin{tabular}{|c|c|c|}
\hline Semigos SAJPPG5 & $\begin{array}{l}\text { Ontros } \\
\text { Estados } \\
\text { Imimplantacio }\end{array}$ & $\begin{array}{c}\text { Ourros } \\
\text { Estados } \\
\text { Pos } \\
\text { Implantacilo }\end{array}$ \\
\hline Configuraçäo dos servidores de borda & $\mathrm{x}$ & \\
\hline Monitoramento os servidores de borda PG & & $\mathrm{x}$ \\
\hline $\begin{array}{l}\text { Acompanhamento da manuten ção dos servidores đe borda e aplica çào } \\
\text { em conjunto com a STI }\end{array}$ & & $\mathbf{x}$ \\
\hline Configuraçäo dos servidores de borda para SG & $\mathrm{x}$ & \\
\hline Configuraçăo, teste e liberação de base EADT & $\mathrm{x}$ & \\
\hline $\begin{array}{l}\text { Configuração, teste e liberaçño de Bases de } \\
\text { Homologaçăo/Treinamento }\end{array}$ & $\mathrm{x}$ & \\
\hline $\begin{array}{l}\text { Visita téenica nas Faculdades para avaliaçào do ambiente de } \\
\text { treinamento para locaçào do espaço c encaminh amento para o } \\
\text { Administrativo }\end{array}$ & $\mathrm{x}$ & \\
\hline
\end{tabular}

Fonte: Softplan (2017).

Com o Controle de Itens de Configuração implementado era possível ter o conhecimento de todos Servidores ativos e operacionais. Caso algum fosse ativado ou desativado, ou alguma configuração de IP ou hostname fosse efetuada, tinha-se um controle diário para gerenciar os Servidores de todas as categorias (aplicação, bordas ou banco de dados), o que favoreceu uma completa verificação do status atual do parque de servidores antes de iniciar o processo de gerenciamento de liberação de versão. Na Figura 5 segue uma das planilhas que compuseram o Controle de Itens de Configuração.

Figura 5 - Parte do Catálogo de Itens de Configuração.

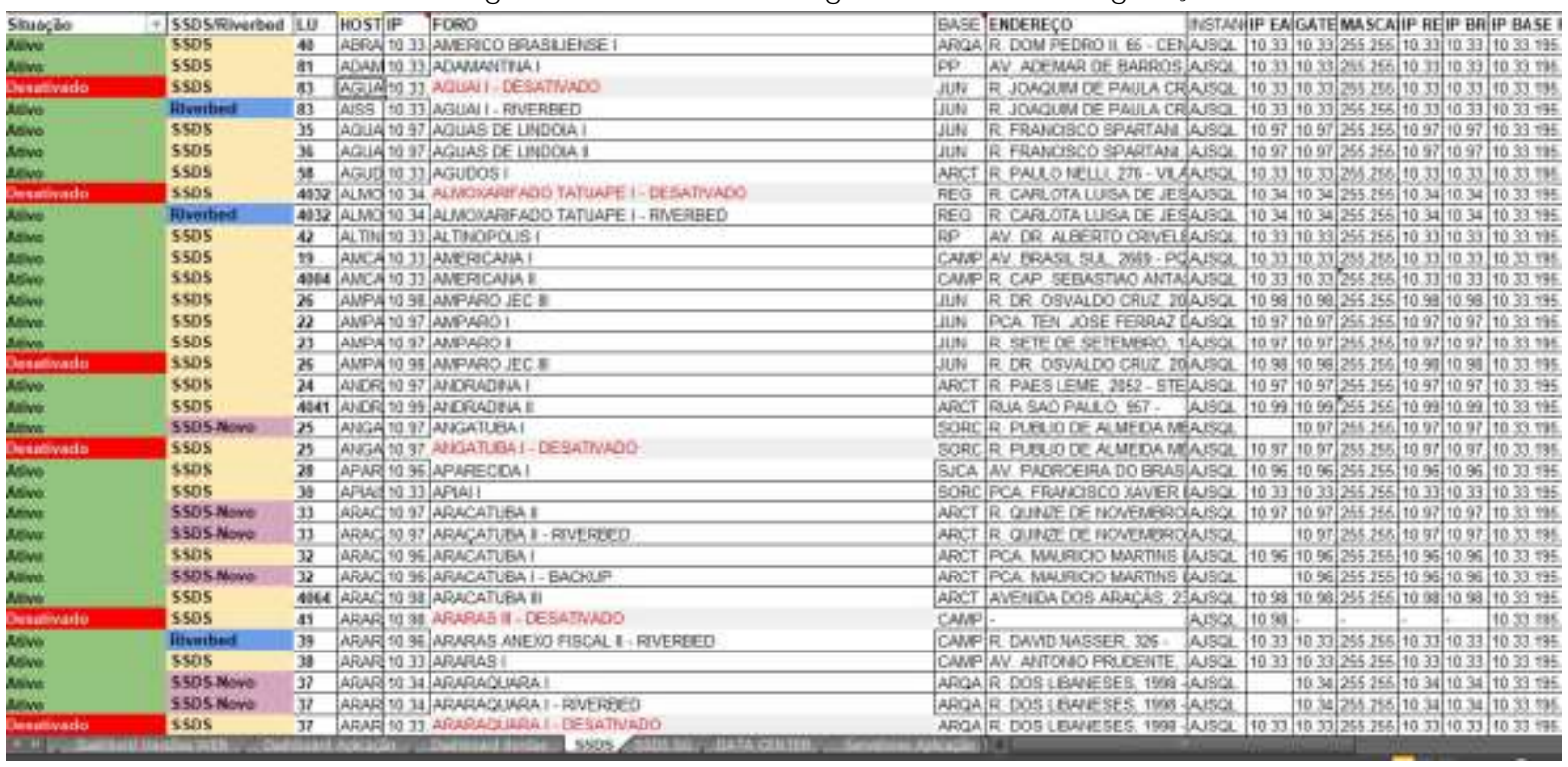

Fonte: Adaptado de Softplan (2017).

Outra recomendação do framework ITIL, é a Matriz de Responsabilidades ou Matriz RACl. Com esta ferramenta foi possível mapear todas as atribuições de cada colaborador para ser usada no momento da execução do processo de gerenciamento de liberação da versão. Independente de qual analista fosse executar as atividades, todos estariam cientes das atribuições e responsabilidades de cada um, proporcionando a execução do processo de forma coordenada e fluente. Na Figura 6 segue a versão da Matriz RACl elaborada. 
Figura 6 - Matriz RACl

\begin{tabular}{|c|c|c|c|c|c|c|c|}
\hline Nalepi & $\angle \mathrm{RACI}$ & & & 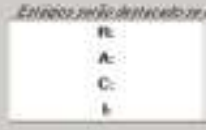 & 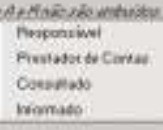 & & \\
\hline \multicolumn{8}{|c|}{ ATIVIDADE Liberaç̄o de Vers̄o } \\
\hline pharrsta & 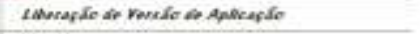 & & & & & & \\
\hline LE-ShimeNTOS & wers & & & & & & \\
\hline ATULLENOD & Enecoua & & & & & & \\
\hline Exitanas : & arechesta & 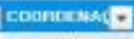 & Fonaturin armene. & 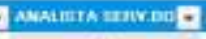 & 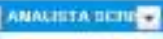 & Masumracin- & Anealinga in \\
\hline 1 & Sesergiode Convas & $\mathrm{CA}$ & Am & c & en & & ' \\
\hline 2 & Niven do secose & cn & $\mathrm{cm}$ & 1 & and & & ' \\
\hline 3 & 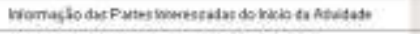 & AIA & 1 & 1 & 1 & 1 & ' \\
\hline+ & 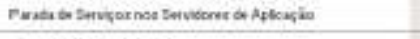 & 1 & Am & 1 & 1 & & 1 \\
\hline s & 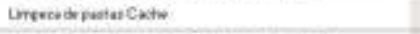 & ' & An & 1 & & & \\
\hline$*$ & 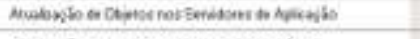 & ca & MR & 1 & 1 & & 1 \\
\hline 7 & 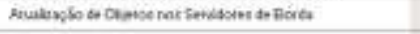 & ᄃл & cal & ant & 1 & 1 & 1 \\
\hline$\cdot$ & Eimeugbotselph & cn & 1 & 1 & $a n$ & cn & ' \\
\hline s & 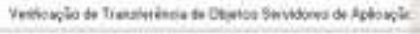 & ch & Ans & 1 & I & 1 & n \\
\hline$n$ & 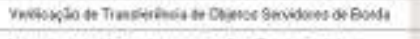 & cin & 1 & An & 1 & 1 & A \\
\hline n & 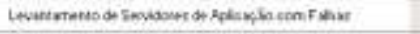 & can & MR & can & & a & म \\
\hline a & 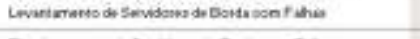 & c & cm & ami & & 1 & $n$ \\
\hline a & 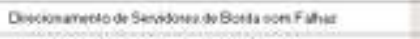 & cn & cn & Am & & 1 & \\
\hline " & 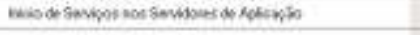 & cin & ANA & 1 & 1 & & 1 \\
\hline n & 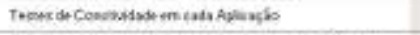 & cil & I & 1 & 1 & & An \\
\hline s & 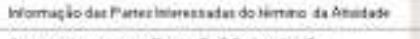 & ant & 1 & 1 & 1 & 1 & ' \\
\hline$n$ & 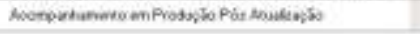 & Ant & A & A & ค & en & A \\
\hline
\end{tabular}

Fonte: Adaptado de Softplan (2017).

Como solução tecnológica proposta, a equipe adotou uma aplicação interna da empresa denominada "Console" o qual já era utilizada em outros clientes, a qual fazia uma leitura do banco de dados, mapeando todos servidores de borda configurados e ativos proporcionando que a distribuição dos objetos de uma pacote de versão fossem distribuídos simultaneamente para todos os servidores de borda, proporcionando uma distribuição uniforme e controlada de forma visual e instantânea, se houve alguma falha na distribuição, ou se houve sucesso na mesma.

De acordo com a definição do framework ITIL, todo processo deve ser mensurável, sendo assim foi necessário a elaboração de métricas e indicadores para avaliar se o processo de gerenciamento de liberação de versão atingiu ou não seu objetivo. Estipularam-se Indicadores-Chave de Desempenho (KPIs), para julgar a eficiência e efetividade do processo.

$\mathrm{Na}$ Figura 7 segue as representações gráficas destes indicadores. Ficaram definidos o registro da data da execução do processo, o no da versão da aplicação, o no de objetos componentes da versão, 0 № de servidores de aplicação envolvidos no processo, assim como o no de servidores de borda. Na sequência, o no dos servidores de aplicação e o $\mathrm{n}$ - dos servidores de borda com falhas du rante o processo de gerenciamento de liberação de versão, o que permitiu a aplicação de soluções de contorno em tempo real, e de forma transparente para o cliente, permitindo que a aplicação fosse atualizada com sucesso em todas estações de trabalho. Por último, os indicadores de sucesso do processo, o no de servidores de aplicação e de bordas com falhas após o processo de gerenciamento de liberação de versão ser finalizado e entregue para o cliente em produção, o qual é demostrado com $100 \%$ de assertividade. 
Figura 7- Controle de KPIs aplicadas no Gerenciamento de Liberação de Versão.

\begin{tabular}{|c|c|c|c|c|c|c|c|c|}
\hline \multirow[b]{2}{*}{ Data } & \multicolumn{8}{|c|}{ Atualização PG } \\
\hline & Versão & Objetos & $\begin{array}{c}\text { Servidores } \\
\text { de } \\
\text { Aplicação }\end{array}$ & \begin{tabular}{|c|}
$\begin{array}{c}\text { Servidores } \\
\text { de Borda }\end{array}$ \\
\end{tabular} & \begin{tabular}{|c|} 
Servidores \\
Aplicação \\
com falhas \\
durante \\
atualização \\
\end{tabular} & \begin{tabular}{|c|} 
Servidores \\
de Borda \\
com falhas \\
durante \\
atualização \\
\end{tabular} & $\begin{array}{c}\text { Servidores } \\
\text { Aplicação } \\
\text { com falhas } \\
\text { após } \\
\text { atualização }\end{array}$ & $\begin{array}{c}\text { Servidores } \\
\text { de Borda } \\
\text { com falhas } \\
\text { após } \\
\text { atualização }\end{array}$ \\
\hline Agosto & 1.5.21-24-A & 28 & 197 & 500 & 0 & 15 & 0 & 0 \\
\hline Agosto & 1.5.21-24-B & 4 & 179 & 0 & 3 & 0 & 0 & 0 \\
\hline Agosto & 1.5.21-24-D & 4 & 179 & 0 & 2 & 0 & 0 & 0 \\
\hline Agosto & $1.5 .21-26$ & 17 & 197 & 500 & 0 & 8 & 0 & 0 \\
\hline Agosto & 1.5.21-26A & 6 & 179 & 500 & 17 & 4 & 0 & $\underline{0}$ \\
\hline Setembro & $1.5 .21-27$ & 18 & 197 & 500 & 0 & 7 & 0 & 0 \\
\hline Setembro & $1.5 .21-29$ & 17 & 197 & 500 & 1 & 11 & 0 & 0 \\
\hline Setembro & 1.5.23-6 & 36 & 197 & 500 & 0 & 4 & 0 & 0 \\
\hline Setembro & 1.5.23-7 & 18 & 197 & 500 & 1 & 4 & 0 & 0 \\
\hline Setembro & 1.5.23-7_B & 7 & 197 & 0 & 0 & 0 & 0 & 0 \\
\hline Outubro & 1.5.23-9 & 20 & 197 & 500 & 0 & 7 & 0 & $\underline{0}$ \\
\hline Outubro & $1.5 .23-9 \mathrm{~A}$ & 2 & 0 & 500 & 0 & 4 & 0 & 0 \\
\hline Outubro & 1.5.23-10 & 20 & 197 & 502 & 0 & 7 & 0 & 0 \\
\hline Outubro & \begin{tabular}{|l}
$1.5 .23-$ \\
$12 / 1.5 .1-0$ \\
\end{tabular} & 24 & 197 & 503 & 0 & 4 & 3 & 0 \\
\hline Novembro & 1.5.23-14 & 17 & 197 & 503 & 0 & 6 & 0 & 0 \\
\hline Novembro & 1.5.23-15 & 18 & 197 & 507 & 0 & 5 & 0 & 0 \\
\hline Novembro & \begin{tabular}{|l|}
$1.5 .24-$ \\
$4 / 1.5 .1-3$ \\
\end{tabular} & 27 & 197 & 507 & 0 & 8 & 0 & 0 \\
\hline Dezembro & 1.5.24-4B & 7 & 191 & 507 & 0 & 1 & 0 & 0 \\
\hline Dezembro & 1.5.24-5A & 22 & 191 & 507 & 0 & 6 & 0 & 0 \\
\hline
\end{tabular}

Fonte: Adaptado de Softplan (2017).

Dentro de seis meses, obteve-se o controle do processo de forma a gerenciá-lo e mensurá-lo, sendo os resultados mostrados ao cliente tanto na qualidade quanto no valor dos serviços, uma vez que o trabalho dos usuários não foi mais afetado por falha de execução do processo gerenciamento de liberação de versão, uma vez que se tornou transparente a eles.

Com o processo documentado e de fluxo definido, todas as partes interessadas no processo de gerenciamento de liberação de versão que a empresa executa, incluído o cliente, apresentaram-se satisfeitos com os resultados obtidos, uma vez que as falhas na transferência dos pacotes de liberação chegaram a zero.

\section{CONCLUSÃo}

Este estudo proporcionou a avaliação da aplicação da metodologia de gerenciamento de processos (BPM) e do framework ITIL aplicados em conjunto, proporcionando resultados de forma a promover o sucesso na execução do processo de gerenciamento de liberação de versão. Pode-se observar a aderência das ferramentas aplicadas, respeitando o contexto da realidade do cenário avaliado.

A pouca exploração em campo da aplicação conjunta da metodologia BPM e o framework ITIL no processo de gerenciamento de liberação de versão em empresas de desenvolvimento de softwarefoi um dos 
principais motivadores para a elaboração deste estudo, uma vez que a liberação de versão representa um processo crítico dentro do contexto de gerenciamento de TI.

0 conhecimento dos colaboradores que passaram a fazer parte da equipe após a reformulação foi fundamental para a correta interpretação e aplicação das práticas apresentadas na metodologia BPM e no framework ITIL no processo de gerenciamento de liberação de versão.

A distribuição das responsabilidades de forma coordenada, fez com que as atividades e suas tarefas pudessem ser executadas de forma organizada, sendo revisada e corrigida em tempo real, antes de finalizar o processo e então liberá-lo em produção. Com o processo então definido e mapeado, foi possível aplicar as ferramentas sugeridas no ITIL de forma assertiva e de fácil compreensão por todas as partes envolvidas, tanto do cliente como da empresa Softplan.

Com os resultados obtidos nesta pesquisa é possível oferecer ao meio acadêmico contribuições ao estudo e aplicação de metodologias e frameworksem conjunto, e o quanto estas podem oferecer na obtenção de resultados mensuráveis às empresas, uma vez que as ferramentas oferecidas poderão ser aplicadas a outras áreas da empresa, seja para o gerenciamento de processos, de pessoas ou de tecnologias.

Como sugestão de trabalhos futuros será interessante promover validações de melhoria contínua sobre os resultados alcançados, uma vez que a área de Tecnologia da Informação está em constante transformação e evolução.

\section{REFERÊNCIAS}

AXELOS. ITIL Maturity Model and Self-Assessment Service: User Guide. Axelos Limited, October, [2013]. Disponível em https://www.axelos.com/Corporate/media/Files/Misc\%20Qualification\%20Docs/ITIL-maturitymodel-self-assessment-service-user-guide.pdf.Acessado em: 21 maio 2017.

BARROS, Marta Duarte de et al. Mapping of the scientific production on the ITIL application published in the national and international literature. Procedia Computer Science, Niterói, Rio de J aneiro, v. 55, p. 102-111, jul. 2015. DOI: 10.1016/j.procs.2015.07.013.

BARROS, Anderson Rocha; SASSI, Renato J osé. Business process management e teo ria das restrições aplicadas nos processos da empresa de Call Center: proposta de melhoria na mediação de reclamações. In: SEMINÁRIOS EM ADMINISTRAÇÃO, 18., 2015, São Paulo. Anais [...]. São Paulo: FEA/USP, 2015. v. 1. p. 1-16.

CALAZANS, Angélica Toffano Seidel; KOSLOSKI, Ricardo Ajax Dias; GUIMARÃES, Fernando de Albuquerque. Proposta de modelo de medições para contratação do gerenciamento de processos de negócio (Business Process Management - BPM). J ISTEM - Journal of Information Systems and Technology Management, Brasília-DF, v. 13, n. 2, p. 275-300, maio/ago. 2016.

CRUZ-HINOJ OSA, Nancy J udith; GUTIÉRREZ-DE-MESA, J osé Antonio. Literature review of the situation research faces in the application of ITIL in small and medium enterprises. Computer Standards \& Interfaces, Madrid, Espanha, v. 48, p. 124-138, maio 2016.

FERNANDES, Agnaldo Aragon; ABREU, Vladmir Ferraz de. Implantando a governança de TI. 2. ed. São Paulo, Brasport, 2014.

GIL, Antônio Carlos. Métodos e técnicas de pesquisa social. 6. ed São Paulo: Atlas, 2008.

GIL-GÓMEZ, Hermenegildo; OLTRA-BADENES, Raúl; ADARME-JAIMES, Wilson. Service Quality Management Based on The Application of The ITIL Standard. Dyna, Medelin, Colômbia, v. 81, n. 186, p. 51-56, ago. 2014.

GOMES, Leonardo Dias; GOULART JÚNIOR, Claudiomar Rrodrigues. Best Practices In Governance Of Information And Tecnology Management. In: CONTECSI, 13., 2016, São Paulo. Anais [...]. São Paulo: FEA/USP, 2016. p. 837-857.

GREAT BRITAIN CABINET OFFICE. ITIL: Service Lifecycle Publication Suite. UK: Editora TSO, 2013. 
LEITE, David Timóteo Carrilho.; SHIBAO, Fábio Ytoshi; FARIA, Ana Cristina de. Gestão de Processos de Negócios Verdes (Green BPM) como ferramenta propulsora de ganhos sustentáveis na gestão de fornecedores de uma metalúrgica de médio porte. In: SEMINÁRIOS DE ADMINISTRAÇÃO, 18., 2015, São Paulo. Anais [...]. São Paulo: FEA/USP, 2018.

LOUREIRO, Leonardo de Castro; PENHA, Thiago de Araújo; NASCIMENTO, J oão Marcos Moura. Relacionamento das Melhores Práticas do Cobit e ITIL para a Governança de TI. In: IX SEGeT 2012, Alagoas. Anais do IX SEGeT, 2012

MOREIRA, J osé Rodrigo Poggio; SILVA, Paulo Caetano. IT Management Model for Financial Report Issuance and Regulatory and Legal Compliance. JISTEM - J ournal of Information Systems and Technology Management, Salvador, Bahia, Brasil, v. 10, n. 3, p. 597-620, set./dez. 2013.

POURMIRZA, Shaya et al. A Systematic Literature Review on The Architecture of Business Process Management Systems. Information Systems, Eindhoven, Holanda, v. 66, p. 43-58, fev. 2017.

RIOS, Orlivaldo Kleber Lima; TEIXEIRA FILHO, J osé Gilson de Almeida; RIOS, Vânia Patrícia da Silva. Melhores práticas do COBIT, ITIL e ISO/IEC 27002 para implantação de política de segurança da informação em Instituições Federais do Ensino Superior. Revista Gestão \& Tecnologia, Pedro Leopoldo, v. 17, n. 1, p. 130153, jan./abr. 2017.

SILVA, J B. Integração entre BPM e ITIL: um estudo de caso na Universidade Federal da Bahia. 2004. Disponível em: https://www.researchgate.net/publication/236143304_Integracao_entre_BPM_e_ITIL-Um_Estudo_de_Caso_na_Universidade_Federal_da_Bahia. Acesso em: 29 maio $2 \overline{0} 17$.

SINCORÁ, Larissa Alves et al. Orientando-se por Processos: iniciativas para a implementação do Business Process Management (BPM). In: SEMINÁRIOS EM ADMINISTRAÇÃO, 17., 2014, São Paulo. Anais [...]. São Paulo: FEA/USP, 2014.

SOFTPLAN. Quem Somos. 2017. Disponível em: https:/www.softplan.com.br/a-softplan/quem-somos/. Acesso em: 21 maio 2017.

VITORIANO, Maria Albeti Vieira; SOUZA NETO, J oão de. Information Technology Service Management Processes Maturity In The Brazilian Federal Direct Administration. JISTEM - Journal of Information Systems and Technology Management, Brasília-DF, Brasil, v. 12, n. 3, p. 663-686, set./dez. 2015.

WAZLAWICK, Raul Sidnei. Uma reflexão sobre a pesquisa em ciência da computação à luz da classificação das ciências e do método científico. Revista de Sistemas de Informação da FSMA, v. 6, p. 3-10, 2010. 